\title{
Transverse nonlinear optics in heavy-metal-oxide glass
}

\author{
Alessia Pasquazi, Salvatore Stivala, and Gaetano Assanto* \\ ${ }^{1}$ NooEL-Nonlinear Optics and OptoElectronics Lab, CNISM, INFN and Department of Electronic Engineering, \\ University "Roma Tre", Via della Vasca Navale 84, 00146 Rome, Italy \\ José Gonzalo and Javier Solis \\ Laser Processing Group, Instituto de Optica, CSIC, Serrano 121, 28006 Madrid, Spain
}

(Received 19 November 2007; published 7 April 2008)

\begin{abstract}
We investigate beam propagation in a heavy-metal-oxide glass of the group $\mathrm{Nb}_{2} \mathrm{O}_{5}-\mathrm{PbO}-\mathrm{GeO}_{2}$ using picosecond pulses in the near infrared. We observe a wavelength-dependent self-focusing, with beam collapse and modulation instability in the Kerr regime at $1.064 \mu \mathrm{m}$ and stable spatial solitons at $820 \mathrm{~nm}$ where multiphoton absorption is present. We report near-infrared beam self-confinement and solitons, filamentation, interactions, and conical emission in the ps regime and discuss them with the aid of a numerical model.
\end{abstract}

DOI: $10.1103 /$ PhysRevA.77.043808

PACS number(s): 42.65.Jx, 42.70.Mp, 42.65.Tg, 42.65.Hw

\section{INTRODUCTION}

Nonlinear glasses have been extensively studied in the past few decades [1-7]. Among them, heavy-metal oxides (HMO) are promising materials for ultrafast photonics, owing to their extended transmittance and large linear $(>2)$ and Kerr nonlinear refractive indices with ultrafast response [1,4-7]. In nonlinear optics of dielectrics, transverse beam effects and-specifically-spatial solitons are some of the most studied and intriguing phenomena, experimentally investigated in media encompassing different physics, from catalytic electronics to photorefraction and molecular reorientation, including plasmas and parametric crystals [8-13]. However, despite the interest in glasses and a conspicuous number of soliton-based applications for all-optical signal processing [14-21], solitary propagation in glass has been reported in a few cases, either with femtosecond pulses in the presence of multiphoton ionization [22-24], or cw excitation in thermo-optic diffusive [25] or photorefractive systems [26]. As compared with the reported experiments in other media, such a limited number of experimental studies can be largely attributed to soliton instability and the tendency to catastrophic collapse in purely Kerr bulk media [27,28], i.e., wherever the response is not mitigated by additional mechanisms such as reduced dimensionality (e.g., planar waveguiding) or saturation or transverse nonlocality or higher order susceptibility $[12,13,29,30]$. Multiphoton absorption, often present when high peak powers are employed, can play a remarkable role in nonlinear optics and in the stabilization of self-confined beams in bulk [31-38].

In this paper we report on nonlinear propagation in $\mathrm{Nb}_{2} \mathrm{O}_{5}-\mathrm{PbO}-\mathrm{GeO}_{2}$ glass, from catastrophic collapse and transverse modulational instability at $1.064 \mu \mathrm{m}$ to single and multiple spatial solitons in the presence of multiphoton absorption at $0.82 \mu \mathrm{m}$. The experiments were carried out with picosecond pulses using both circular and elliptical transverse excitations over a set of propagation distances and energies.

\footnotetext{
*assanto@uniroma3.it; URL: http://optow.ele.uniroma3.it/
}

\section{MATERIAL PROPERTIES AND EXPERIMENTAL SETUP}

HMO are characterized by phonon energies significantly lower than in silicate and borate glasses, although slightly higher than fluoride and chalcogenide systems; they are transparent and maintain good mechanical properties over a wide interval of wavelengths from the visible up to the mid-ir $(7-8 \mu \mathrm{m})[1,4]$.

$\mathrm{HMO}$ glasses $25 \mathrm{Nb}_{2} \mathrm{O}_{5}-25 \mathrm{PbO}-50 \mathrm{GeO}_{2}$ mol \% (nominal composition) were obtained by mixing high-purity reagents and employing standard melting procedures. The mixture was prepared in a platinum crucible and kept in a furnace at temperatures of $1100-1300{ }^{\circ} \mathrm{C}$ for one hour and then poured on a brass plate; this was followed by thermal annealing for one hour at $450{ }^{\circ} \mathrm{C}$ and by slow cooling to room temperature at $1.5{ }^{\circ} \mathrm{C} / \mathrm{min}$. The resulting material was a transparent glass with a yellowish appeareance at the eye. The glass was subsequently cut in blocks with parallel faces and polished to optical grade. HMO transmittance and refractive index were determined by spectroscopic ellipsometry [39], as graphed in Fig. 1 after correction for reflection (Fresnel) losses.

For the nonlinear characterization, a HMO sample of dimensions $5.7 \times 8.7 \times 11.2 \mathrm{~mm}$ was placed in front of the 25 ps pulsed beam produced by a $10 \mathrm{~Hz}$ repetition-rate parametric generator (OPG), tunable from 0.72 to $2.1 \mu \mathrm{m}$, seeded by a parametric oscillator and synchronously pumped by an amplified frequency-doubled Nd:YAG laser. The beam was

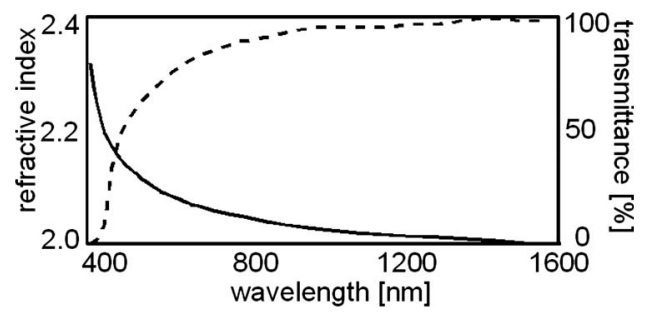

FIG. 1. Refractive index (solid line) and linear transmittance (corrected for Fresnel losses) (dashed line) of HMO vs wavelength, as obtained by spectroscopic ellipsometry. 


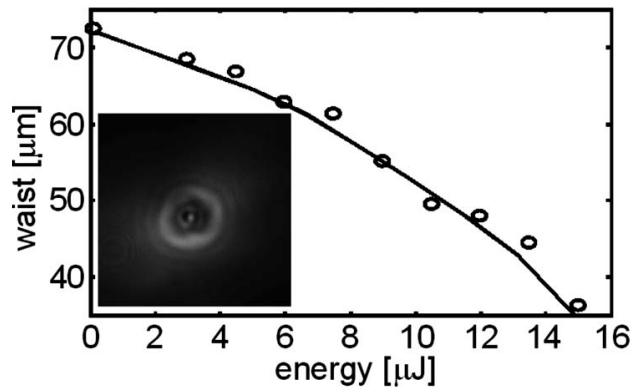

FIG. 2. Measured (circle) and numerically evaluated output beam size vs input energy, for a beam launched with waist $18 \mu \mathrm{m}$ and propagating over $5.7 \mathrm{~mm}$ at $\lambda=1064 \mathrm{~nm}$. The inset shows a case of optical damage induced by pulses of energy $>16 \mu \mathrm{J}$.

spatially filtered to the fundamental $\mathrm{TEM}_{00}$ mode, characterized and monitored with an automated profiler and gently focused on the input facet of the sample. A $35 \mathrm{~mm}$ lens was used to in-couple circularly symmetric beams for soliton excitation, whereas a pair of cylindrical lenses with focal lengths $f=15$ and $f=100 \mathrm{~mm}$, respectively, was employed to shape the elongated (elliptical) input for the study of modulational instability. Polarizing optics and half-wave plates allowed adjustment of both power and polarization. Images of the output beam were collected by an infrared-enhanced charge-coupled device (CCD) camera through a microscope objective, while calibrated semiconductor photodiodes measured input and transmitted power and energy with the aid of beam splitters. A dual channel boxcar averager and computer control were used to filter out noise as well as pulses of amplitude exceeding the prescribed interval of values.

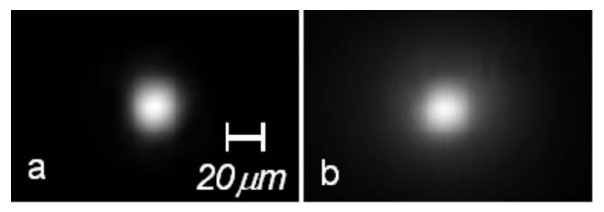

FIG. 3. Self-confinement at $\lambda=820 \mathrm{~nm}$ : (a) input and (b) output intensity profiles of a high-power pulsed beam launched with a waist of $11 \mu \mathrm{m}$ and propagating over $5.7 \mathrm{~mm}$. The beam size remains unchanged for more than 6 Rayleigh lengths, demonstrating the formation of a stable solitonlike beam.

\section{EXPERIMENTS AND DATA REDUCTION}

\section{A. Model}

Laser beam propagation in optical dielectrics with a Kerr response and dissipation can be described by a nonlinear Schrödinger equation (1) corrected for (multiphoton) absorption,

$$
2 i k \partial A_{z}+\nabla_{\perp}^{2} A+\frac{n_{2} k^{2}}{\eta_{0}}|A|^{2} A+i k \beta_{K}\left(\frac{n_{0}}{2 \eta_{0}}\right)^{K-1}|A|^{2 K-2} A=0
$$

with $A$ being the slowly varying amplitude of the electric field $E(x, y, z, t)=\frac{1}{2} A(x, y, z, t) \exp [i(k z-\omega t)]+c . c ., \quad k$ the wave number, $\eta_{0}$ the vacuum impedance, $n_{0}$ the refractive index, and $n_{2}$ the Kerr coefficient as in $n(I)=n_{0}+n_{2} I . \beta_{K}$ is the coefficient of $K$-photon absorption as defined by $\partial I_{z}$ $=-\beta_{K} I^{K}$. For the numerics we employed a $(2 D+1)$ beam

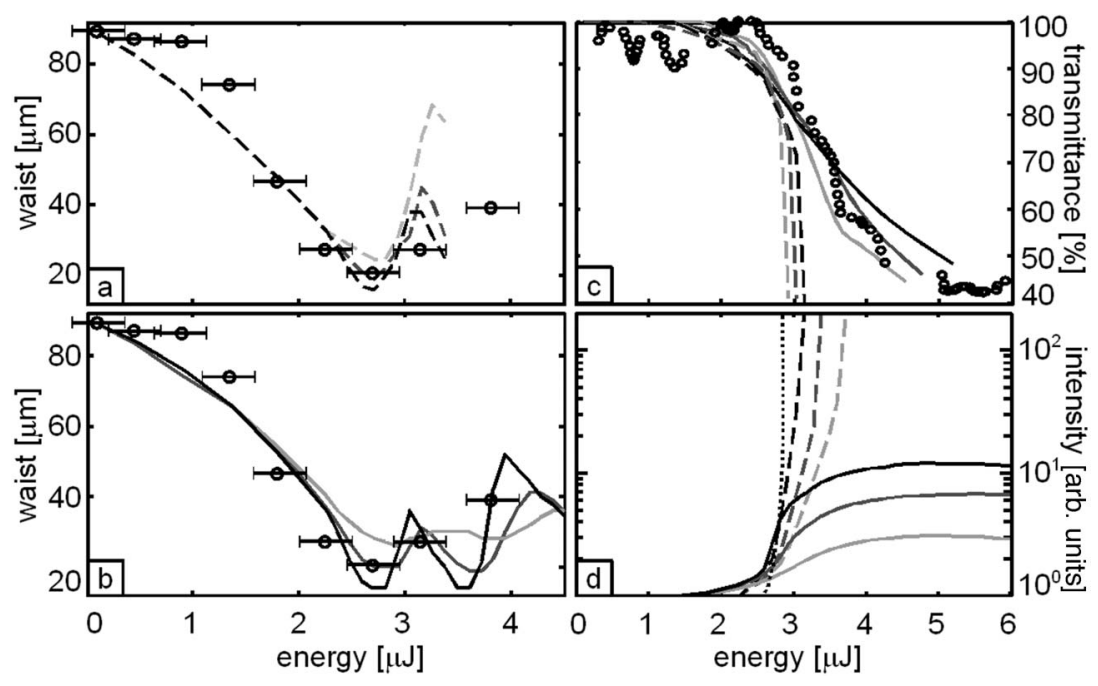

FIG. 4. Summary of experimental results and numerical evaluations for an $11 \mu \mathrm{m}$ circular input at $\lambda=820 \mathrm{~nm}$. [(a) and (b) $]$ Output beam waist (FWHM) vs excitation energy for propagation over $5.7 \mathrm{~mm}$. Data (open circles) and calculation for a Kerr coefficient $n_{2}=5.5$ $\times 10^{-15} \mathrm{~cm}^{2} / \mathrm{W}$ in the presence of either (a) two-photon absorption (2PA, dashed lines) or (b) three-photon absorption (3PA, solid lines). The time integration was implemented for input Gaussian pulses. The $2 \mathrm{PA}$ coefficients are $1.2,1.8,2.4 \times 10^{-2} \mathrm{~cm} / \mathrm{GW}$ and the $3 \mathrm{PA}$ coefficients are 1.5, 3.0, $7 \times 10^{-4} \mathrm{~cm}^{3} / \mathrm{GW}^{2}$, respectively, with darker lines referring to lower absorption. Best fits are obtained for $\beta_{2}$ $=1.8 \times 10^{-2} \mathrm{~cm} / \mathrm{GW}$ and $\beta_{3}=3 \times 10^{-4} \mathrm{~cm}^{3} / \mathrm{GW}^{2}$ in the two cases. (c) Beam transmission through an $11.2 \mathrm{~mm}$ sample vs input energy: experimental (open circles) and time integrated data calculated in the presence of 2PA (dashed lines) and 3PA (solid lines). The best fit is obtained considering 3PA with $\beta_{3}=3 \times 10^{-4} \mathrm{~cm}^{3} / \mathrm{GW}^{2}$. (d) Computed highest (along $z$ ) intensity of the pulse peak vs excitation for pure Kerr (dotted black line) with no $K$-photon absorption, Kerr with 2PA (dashed lines), and Kerr with 3PA (solid lines), using the coefficients and the gray scale above: in the model only 3PA can effectively counteract collapse in the whole excitation range. 
propagator with a standard Crank-Nicolson scheme and Gaussian spatiotemporal excitation.

\section{B. Self-focusing and spatial solitons}

When excited in the nonlinear regime at $1064 \mathrm{~nm}$ with a beam of waist $18 \mu \mathrm{m}$, the HMO glass behaved as a purely Kerr medium and exhibited self-focusing. Although the output spot size decreased with excitation (Fig. 2), spatial solitons of size comparable to the input waist were not observed and, for pulse energies of $16 \mu \mathrm{J}$ and beyond, the beam collapsed and damaged the sample, visually resulting in a small and faint central spot surrounded by an annular ring as in the inset of Fig. 2. Such an output profile could also be imaged by launching a low-power beam in the damaged region of the sample, confirming the permanent character of the effect. The damage and its dynamics can be ascribed to plasmainduced avalanche breakdown for self-focused picosecond pulses in bulk solids [40,41]. Specifically in HMO glasses, catastrophic self-focusing involves a collateral stress-induced material densification (bright halo region in the inset) due to the high pressure generated in the collapsing region [42]. In order to evaluate the nonlinear refractive index we numerically fitted the data on output beam size [full width at half maximum (FWHM)] versus energy by solving Eq. (1) with $\beta_{K}=0$. We obtained $n_{2}=1.6 \times 10^{-15} \mathrm{~cm}^{2} / \mathrm{W}$, corresponding to a critical power $P_{C R}=\lambda^{2} / 2 \pi n_{0} n_{2} \approx 560 \mathrm{~kW}$ [43]; Fig. 2 graphs both measured and evaluated output waist (FWHM) versus excitation.

Conversely, when operating at $\lambda=820 \mathrm{~nm}$ stable solitary beams could be excited with input waists of $11 \mu \mathrm{m}$ at energies of $2.8 \mu \mathrm{J}$ as visible in Fig. 3, showing intensity profiles at the input and after propagation for $5.7 \mathrm{~mm}$ (6 Rayleigh lengths). The nonlinear absorption amounted to a loss of about $20 \%$, but the sample was not damaged for energies up to $5.4 \mu \mathrm{J}$. The different behavior at the two wavelengths (820 and $1064 \mathrm{~nm}$ ) can be attributed to the presence of multiphoton absorption at $820 \mathrm{~nm}$, as revealed by the throughput reduction, displayed in Fig. 4(c) for an $11 \mu \mathrm{m}$ beam propagating over a length of $11.2 \mathrm{~mm}$ (open circles). The data are normalized to the linear transmittance (which accounts for Fresnel losses).

Owing to the use of picosecond pulses, higher-order dynamics such as group velocity dispersion and plasmainduced defocusing can be neglected. Plasma formation in self-confined propagation of ps pulses, in fact, would be ac-

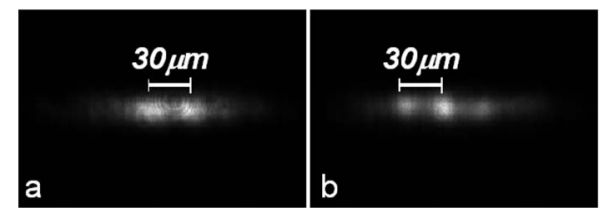

FIG. 5. Output intensity distribution for an elliptically shaped beam propagating through $5.7 \mathrm{~mm}$ at $\lambda=1064 \mathrm{~nm}$. The sample was excited with $0.14 \mathrm{~mJ}$ per pulse and spot size $15 \mu \mathrm{m} \times 215 \mu \mathrm{m}$ in two distinct locations at the entrance facet: either two or three filaments were generated with a separation of $30 \mu \mathrm{m}$.

companied by breakdown, as reported in fused silica [40,41]: once the plasma density is high enough to arrest selffocusing (about $10^{18} \mathrm{~cm}^{-3}$ [41]), strong avalanche ionization takes place with an amplified electron density up to the damage concentration $\left(>10^{19} \mathrm{~cm}^{-3}\right)$ [44]. With femtosecond pulses, conversely, their short duration prevents avalanche multiplication and allows self-trapping without collapse [22].

Two and three-photon energies in bulk HMO at $\lambda$ $=820 \mathrm{~nm}$ are close to 3.02 and $4.54 \mathrm{eV}$, respectively. However, due to the mixed valence states, the glass sample does not exhibit a sharp cutoff in transmittance, as in other HMO glasses [45]. For this reason the evaluation of energy gap and Urbach tail [46] can be affected by a significant error; hence, we resorted to numerical simulations to identify the leading contribution of nonlinear absorption. A summary of the fits with 2PA or 3PA is presented in Fig. 4. Consistently with the dispersion due to the blue-ultraviolet resonance [47], the extrapolated Kerr coefficient $n_{2}=5.5 \times 10^{-15} \mathrm{~cm}^{2} / \mathrm{W}$ at 820 $\mathrm{nm}$ is higher than at $1064 \mathrm{~nm}$. Figure 4 shows the experimental results and those calculated with the inclusion of 2PA [panel (a)] or 3PA [panel (b)] for the output beam waist versus input energy after propagation over $5.7 \mathrm{~mm}$. Although a pointwise interpolation could be obtained using 2PA at given powers, the 3PA term in Eq. (1) allows to fit also the transmittance curve [Fig. 4(c)] and to reproduce the experimental trend in a noncritical fashion, as displayed in Fig. 4(d). Here the highest beam intensity for the pulse peak power is calculated versus input energy at the focal position during propagation in the whole sample, for the pure Kerr case (without $K$-photon absorption) and in the presence of either 2PA or 3PA. The graphs are normalized to the peak intensity at the input; hence, in the diffracting regime (below the soliton threshold $2.8 \mu \mathrm{J}$ ) the highest beam intensity occurs at the entrance (unitary response); at threshold it is at
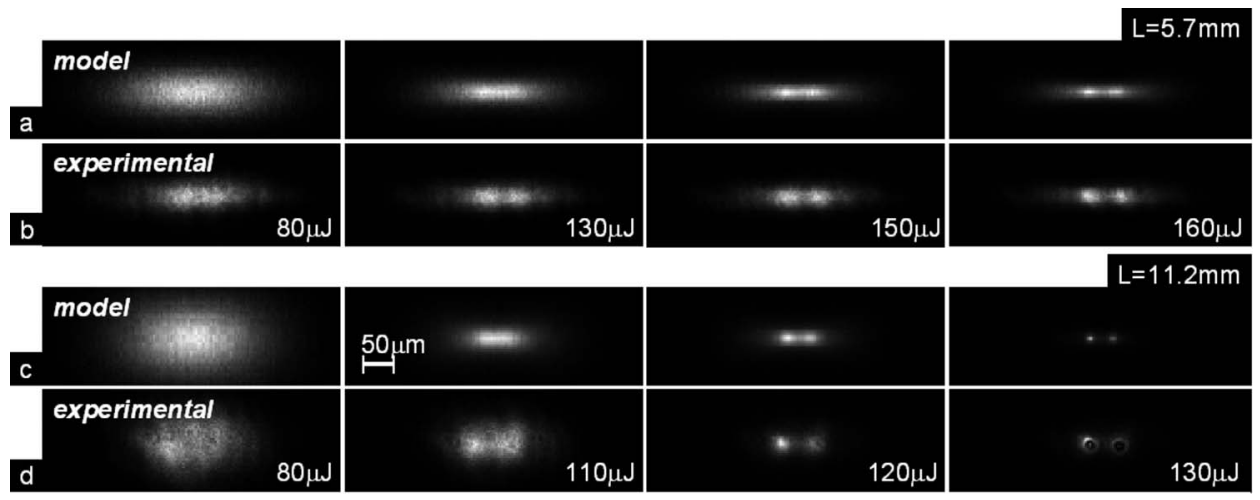

$\mathrm{L}=11.2 \mathrm{~mm}$

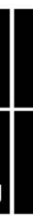

FIG. 6. Propagation of an elliptically shaped beam launched at $\lambda=1064 \mathrm{~nm}$ with size $15 \mu \mathrm{m}$ $\times 215 \mu \mathrm{m}$ : output profiles for propagation lengths of $[$ (a) and (b)] $5.7 \mathrm{~mm}$ and [(c) and (d)] 11.2 $\mathrm{mm}$; (b) and (d) are the experimental results, and (a) and (c) are numerical simulations with $n_{2}$ $=1.6 \times 10^{-15} \mathrm{~cm}^{2} / \mathrm{W}$. 
the output and the response is comparable for 2PA and 3PA; above $2.8 \mu \mathrm{J}$ the focal point moves toward the entrance as the excitation increases. The diverging trend of the maximum peak intensity in the case of $2 \mathrm{PA}$ at energies $>2.8 \mu \mathrm{J}$ indicates the occurrence of catastrophic collapse, never observed at this wavelength. Conversely, 3PA appears to counteract the self-focusing instability in the whole excitation range, with a best fit obtained for $\beta_{3}=3 \times 10^{-4} \mathrm{~cm}^{3} / \mathrm{GW}^{2}$.

\section{Modulational instability and multiple filamentation}

Modulation instability (MI) is a precursor of individual and multiple soliton formation $[10,11,48]$ : the interplay between nonlinearity and diffraction can break up a homogeneous beam into localized filaments, eventually generating an array of solitons [49-61]. The unstable behavior observed at $1064 \mathrm{~nm}$ was confirmed by investigating MI of a beam of size $15 \mu \mathrm{m} \times 215 \mu \mathrm{m}$ injected at the input of the HMO sample. By letting the beam propagate through the three distinct lengths available in our glass block, we recorded the formation of filaments along the major axis of the ellipse, with patterns determined by both input position and propagation distance. Consistently with the theory [49], even when the number of filaments changed owing to a localized perturbation (i.e., launching the beam at a specific input location), for a given excitation energy the spacing between filaments was conserved, as shown in Fig. 5. Filaments catastrophically collapsed for energies higher than $160 \mu \mathrm{J}$ when propagating through the shortest sample section and than $130 \mu \mathrm{J}$ through the longest one. Simulations performed with the model used for propagation at $1064 \mathrm{~nm}$, adding white noise (10\% of beam amplitude) to the input field result in good agreement with the experimental data, as shown in Fig. 6.

Although 3PA plays the role of stabilizing mechanism for spatial solitons at $820 \mathrm{~nm}$, it necessarily shortens the useful length for self-trapped propagation. This limitation can be mitigated by a surrounding energy background, the latter able to "refill" the energy lost by the beam(s) $[32,62]$. We numerically investigated such nonlinear space dynamics in

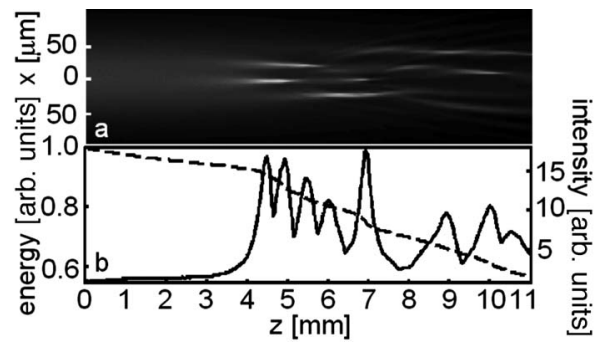

FIG. 7. (a) Intensity distribution calculated in the plane $x z$ for an elliptical beam at $820 \mathrm{~nm}$ launched with size $13.5 \mu \mathrm{m} \times 130 \mu \mathrm{m}$ with energy $24 \mu \mathrm{J}$ assuming $n_{2}=5.5 \times 10^{-15} \mathrm{~cm}^{2} / \mathrm{W}$ and $\beta_{3}=3$ $\times 10^{-4} \mathrm{~cm}^{3} / \mathrm{GW}^{2}$. As it propagates from left to right, the beam undergoes multiple filamentation with conical emission, refocusing as well as formation of new filaments. (b) Input-normalized energy (dashed line) and maximum transverse intensity (solid line) vs propagation.

those conditions under which MI and multiple filamentation were observed in HMO. The resulting evolution pattern, presented in Fig. 7(a), suggests that the propagating filaments undergo complex phenomena such as multiple focusing (breathing) with conical emission from the foci and additional filament generation from the released energy $[63,64]$. The beam was launched from the left and superimposed to a white noise pattern to permit the (numerical) development of MI [49]. Figure 7(b) shows the energy evolution (dashed line) and the corresponding maximum transverse peak intensity (solid line) versus $z$ : the peak value increases by more than one order of magnitude with respect to the initial sections where filaments are yet to be formed. The energy trend demonstrates that the role of nonlinear absorption becomes more appreciable (i.e., steeper decrease vs $z$ ) once selffocusing comes into play. Most filament energy is released after collapse into "spatial" (as compared to spatiotemporal) conical emission, as previously observed also in other nondissipative media $[63,65,66]$, notwithstanding the role of multiphoton losses in transforming the Gaussian excitation into a Bessel-type beam $[32,62,67]$. Finally, the radiated en-
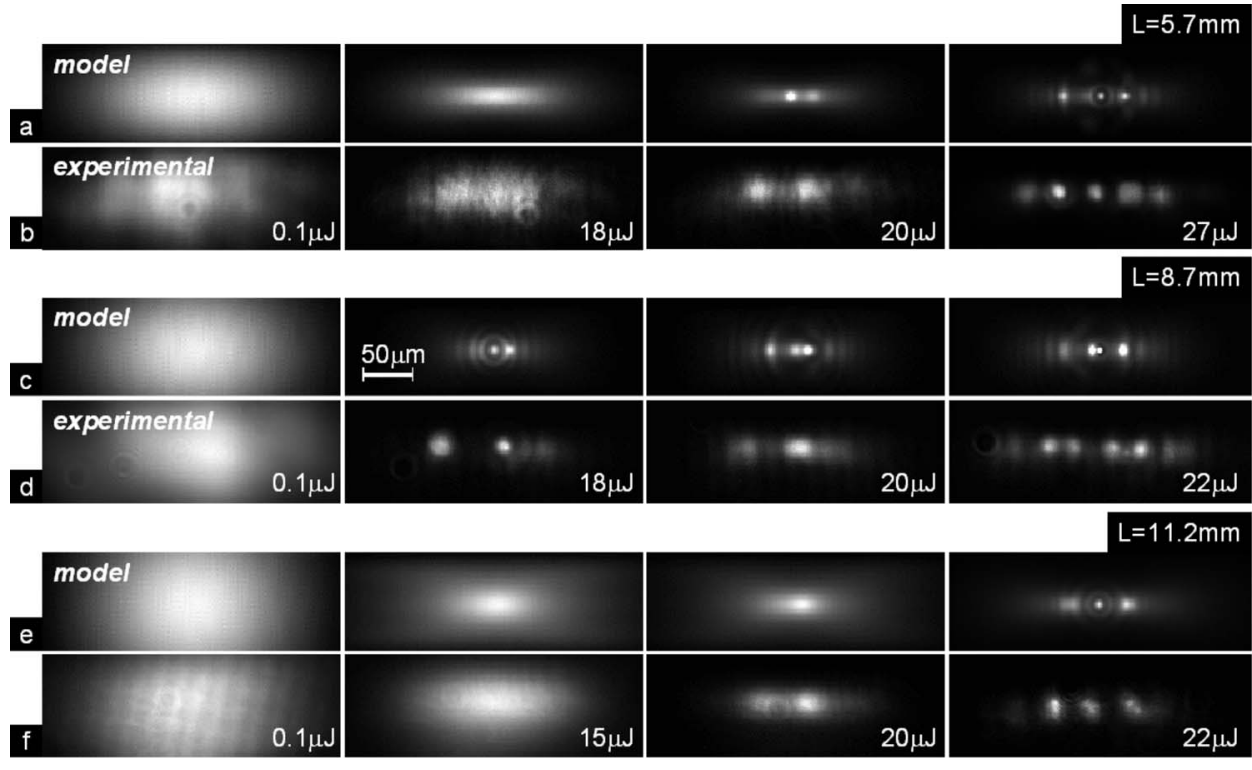

$L=11.2 \mathrm{~mm}$

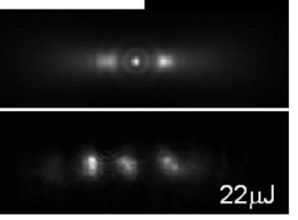

FIG. 8. (a) Calculated and (b) acquired output cross sections of elliptic beams launched at $\lambda$ $=820 \mathrm{~nm}$ with size $13.5 \mu \mathrm{m}$ $\times 130 \mu \mathrm{m}$ after propagation over $5.7 \mathrm{~mm}$. [(c) and (d)] As (a) and (b) but for propagation over 8.7 mm. [(e) and (f)] As (a) and (b) but for input size $13.5 \mu \mathrm{m}$ $\times 180 \mu \mathrm{m}$ and propagation over $11.2 \mathrm{~mm}$. In the numerics we used $n_{2}=5.5 \times 10^{-15} \mathrm{~cm}^{2} / \mathrm{W}$ and $\beta_{3}$ $=3 \times 10^{-4} \mathrm{~cm}^{3} / \mathrm{GW}^{2}$. 


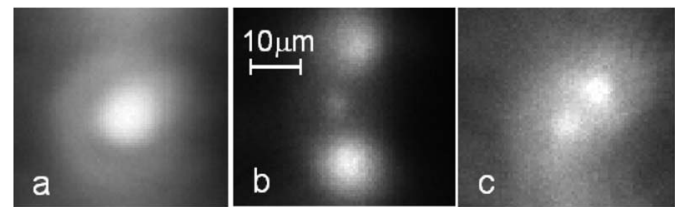

FIG. 9. Details on the filamentation of elliptic beams at $\lambda$ $=820 \mathrm{~nm}$ : (a) for input energy of $27 \mu \mathrm{J}$ and propagation through a distance of $5.7 \mathrm{~mm}$; (b) energy $22 \mu \mathrm{J}$ and distance $8.7 \mathrm{~mm}$; (c) energy $22 \mu \mathrm{J}$ and distance $11.2 \mathrm{~mm}$.

ergy could seed a successive generation of filaments as in Ref. [63].

In the experiments we focused an elliptically shaped Gaussian beam of dimensions $13.5 \mu \mathrm{m} \times 130 \mu \mathrm{m}$ at the input facet of the HMO sample; at variance with the $1064 \mathrm{~nm}$ excitation, the generated filaments did not undergo collapse or damage but their number increased with input energy, in agreement with the predictions from standard treatments of transverse modulational instability [49]. Figure 8(b) shows the output intensity profiles after propagation for $5.7 \mathrm{~mm}$ : two and five filaments were formed at 20 and $27 \mu \mathrm{J}$, respectively. In the latter case, small rings appeared around the beam axes owing to energy release via conical emission [details in Fig. 9(a)]. Moreover, consistently with their predicted evolution (Fig. 7), the filaments exhibited various sizes and intensities due to maximum self-focusing at different propagation distances. The calculated profiles [Fig. 8(a)], obtained with the actual input parameters, are in remarkable agreement with the observed dynamics: self-focusing at low energies, breakup and splitting in two filaments, conical emission, etc. even though their transverse shape and locations depend on the specific realization of a process (MI and multiple filamentation) which is seeded by noise. While the inclusion of 3PA allowed us to reproduce with remarkable fidelity the observed behavior even for longer propagation distances [e.g., 8.7 and $11.2 \mathrm{~mm}$, Figs. 8(c) and 8(d) and
Figs. 8(e) and 8(f), respectively], no agreement was found with the experimental results when excluding 3PA from the model [Eq. (1)] and accounting for 2PA only. This further confirms that three-photon absorption is the leading dissipative mechanism at $820 \mathrm{~nm}$ and the one responsible for soliton stabilization in this HMO.

\section{CONCLUSIONS}

In conclusion, we thoroughly investigated the nonlinear response of a heavy-metal-oxide glass of the ternary system $\mathrm{Nb}_{2} \mathrm{O}_{5} \mathrm{GeO}_{2} \mathrm{PbO}$ when spatially self-focusing ps beams in the near infrared. The results, interpreted with the aid of a nonlinear Schrödinger equation corrected for multiphoton absorption, indicate that the glass has a purely Kerr response around $1064 \mathrm{~nm}$, whereas three-photon absorption plays a significant role around $820 \mathrm{~nm}$. At the latter wavewlength 3PA prevents catastrophic collapse and allows the formation of stable spatial solitary waves, even when originated from a wide beam through modulational instability. Complex dynamics such as conical emission and multiple filamentation were observed and numerically reproduced, confirming the Kerr-type nature of the HMO response. This HMO glass appears to be an excellent candidate for self-confined propagation in ultrafast dielectrics.

\section{ACKNOWLEDGMENTS}

The authors are grateful to J. M. Fernandez-Navarro (Ist. de Optica) for the samples, to C. Afonso (Ist. de Optica), and L. Colace (NooEL) for enlightening discussions. This work was funded by the Italian Ministry for University and Research through an "Italy-Spain Integrated Action" (Grant No. IT1890/HI2006-0095,A.F.2007) and by the Spanish Ministry of Education and Science (Grants No. MAT2005-06508C02-01 and No. TEC2005-00074/MIC).
[1] M. K. T. Clement, J. S. Hayden, Y. T. Hayden, H.-H. Hoffman, F. T. Lentes, and N. Neuroth, in The Properties of Optical Glass, edited by M. Bach and N. Neuroth (Springer-Verlag, Berlin, 1995), pp 19-165.

[2] G. Assanto, A. Gabel, C. T. Seaton, G. I. Stegeman, C. Ironside, and T. J. Cullen, Electron. Lett. 23, 484 (1987).

[3] K. W. DeLong, A. Gabel, C. T. Seaton, and G. I. Stegeman, J. Opt. Soc. Am. B 6, 1306 (1989).

[4] M. E. Lines, J. Appl. Phys. 69, 6876 (1991).

[5] I. Kang, T. D. Krauss, F. W. Wise, B. G. Aitken, and N. F. Borrelli, J. Opt. Soc. Am. B 12, 2053 (1995).

[6] C. B. de Araujo, E. L. Falcao-Filho, A. Humeau, D. Guichaoua, G. Boudebs, and L. R. P. Kassab, Appl. Phys. Lett. 87, 221904 (2005).

[7] J. Gonzalo, H. Fernandez, J. Solis, D. Munoz-Martin, J. M. Fernandez-Navarro, C. N. Afonso, and J. L. G. Fierro, Appl. Phys. Lett. 90, 251907 (2007).

[8] G. Assanto and G. I. Stegeman, Opt. Express 10, 388 (2002).
[9] E. V. Vanin, A. I. Korytin, A. M. Sergeev, D. Anderson, M. Lisak, and L. Vázquez, Phys. Rev. A 49, 2806 (1994).

[10] S. Trillo and W. E. Torruellas, Spatial Solitons (SpringerVerlag, Berlin, Germany, 2001).

[11] Y. S. Kivshar and G. P. Agrawal, Optical Solitons (Academic Press, San Diego, USA, 2003).

[12] C. Conti and G. Assanto, in Encyclopedia of Modern Optics, edited by R. D. Guenther, D. G. Steel, and L. Bayvel (Elsevier, Oxford, UK, 2004), Vol. 5, 43-45.

[13] G. Stegeman, D. N. Christodoulides, and M. Segev, IEEE J. Sel. Top. Quantum Electron. 6, 1419 (2000).

[14] Y. S. Kivshar and G. I. Stegeman, Opt. Photonics News 13, 59 (2002).

[15] M. Peccianti, C. Conti, G. Assanto, A. DeLuca, and C. Umeton, Appl. Phys. Lett. 81, 3335 (2002).

[16] M. Peccianti, A. Dyadyusha, M. Kaczmarek, and G. Assanto, Nat. Phys. 2, 737 (2006).

[17] P. V. Mamyshev, A. Villeneuve, G. I. Stegeman, and J. S. 
Aitchison, Electron. Lett. 30, 726 (1994).

[18] L. Friedrich, G. I. Stegeman, P. Millar, C. J. Hamilton, and J. S. Aitchison, Opt. Lett. 23, 1438 (1998).

[19] M. Shalaby and A. Barthelemy, Opt. Lett. 16, 1472 (1991).

[20] W. Krolikowski and Y. S. Kivshar, J. Opt. Soc. Am. B 13, 876 (1996).

[21] A. Fratalocchi, G. Assanto, K. A. Brzdakiewicz, and M. A. Karpierz, Appl. Phys. Lett. 86, 051112 (2005).

[22] S. Tzortzakis, L. Sudrie, M. Franco, B. Prade, A. Mysyrowicz, A. Couairon, and L. Bergé, Phys. Rev. Lett. 87, 213902 (2001).

[23] L. Sudrie, A. Couairon, M. Franco, B. Lamouroux, B. Prade, S. Tzortzakis, and A. Mysyrowicz, Phys. Rev. Lett. 89, 186601 (2002).

[24] S. Tzortzakis, D. G. Papazoglou, and I. Zergioti, Opt. Lett. 31, 796 (2006).

[25] C. Rotschild, O. Cohen, O. Manela, M. Segev, and T. Carmon, Phys. Rev. Lett. 95, 213904 (2005).

[26] Z. Chen, M. Asaro, O. Ostroverkhova, W. E. Moerner, M. He, and R. J. Twieg, Opt. Lett. 28, 2509 (2003).

[27] R. Y. Chiao, E. Garmire, and C. H. Townes, Phys. Rev. Lett. 13, 479 (1964).

[28] P. L. Kelley, Phys. Rev. Lett. 15, 1005 (1965).

[29] C. Conti, M. Peccianti, and G. Assanto, Phys. Rev. Lett. 91, 073901 (2003).

[30] N. N. Akhmediev and A. Ankiewicz, Solitons: Nonlinear Pulses and Beams (Chapman Hall, London, UK, 1997).

[31] K. W. DeLong, K. B. Rochford, and G. I. Stegeman, Appl. Phys. Lett. 55, 1823 (1989).

[32] A. Dubietis, E. Gaizauskas, G. Tamosauskas, and P. Di Trapani, Phys. Rev. Lett. 92, 253903 (2004).

[33] H. S. Eisenberg, R. Morandotti, Y. Silberberg, S. Bar-Ad, D. Ross, and J. S. Aitchison, Phys. Rev. Lett. 87, 043902 (2001).

[34] J. S. Aitchison, D. C. Hutchings, J. U. Kang, G. I. Stegeman, and A. Villeneuve, IEEE J. Quantum Electron. 33, 341 (1997).

[35] D. C. Hutchings, J. S. Aitchison, and J. M. Arnold, J. Opt. Soc. Am. B 14, 869 (1997).

[36] A. S. Rodrigues, M. Santagiustina, and E. M. Wright, Opt. Quantum Electron. 29, 961 (1997).

[37] A. N. Belanger, A. Villeneuve, and J. S. Aitchison, J. Opt. Soc. Am. B 14, 3003 (1997).

[38] A. Pasquazi, S. Stivala, G. Assanto, J. Gonzalo, J. Solis, and C. N. Afonso, Opt. Lett. 32, 2103 (2007).

[39] J. Gonzalo, O. Sanz, A. Perea, J. M. Fernandez-Navarro, C. N. Afonso, and J. Garcia-Lopez, Appl. Phys. A: Mater. Sci. Process. 76, 943 (2003).

[40] E. Yablonovitch and N. Bloembergen, Phys. Rev. Lett. 29, 907 (1972).

[41] N. Bloembergen, IEEE J. Quantum Electron. 10, 375 (1974).

[42] J. Siegel, J. M. Fernandez-Navarro, A. Garcia-Navarro, V. Diez-Blanco, O. Sanz, J. Solis, F. Vega, and J. Armengol,
Appl. Phys. Lett. 86, 121109 (2005).

[43] G. Fibich and A. L. Gaeta, Opt. Lett. 25, 335 (2000).

[44] B. C. Stuart, M. D. Feit, S. Herman, A. M. Rubenchik, B. W. Shore, and M. D. Perry, Phys. Rev. B 53, 1749 (1996).

[45] X. Liu, D. B. Hollis, and J. McDougall, Phys. Chem. Glasses 37, 160 (1996).

[46] K. Saito and A. J. Ikushima, Phys. Rev. B 62, 8584 (2000).

[47] R. DeSalvo, A. A. Said, D. J. Hagan, E. W. VanStryland, and M. Sheik-Bahae, IEEE J. Quantum Electron. 32, 1324 (1996).

[48] A. J. Campillo, S. L. Shapiro, and B. R. Suydam, Appl. Phys. Lett. 23, 628 (1973).

[49] V. I. Bespalov and V. I. Talanov, JETP Lett. 3, 307 (1966).

[50] R. A. Fuerst, D. M. Baboiu, B. Lawrence, W. E. Torruellas, G. I. Stegeman, S. Trillo, and S. Wabnitz, Phys. Rev. Lett. 78, 2756 (1997).

[51] H. Fang, R. Malendevich, R. Schiek, and G. I. Stegeman, Opt. Lett. 25, 1786 (2000).

[52] D. Kip, M. Soljajic, M. Segev, E. Eugenieva, and D. N. Christodoulides, Science 290, 495 (2000).

[53] R. Malendevich, L. Jankovic, G. Stegeman, and J. S. Aitchison, Opt. Lett. 26, 1879 (2001).

[54] R. Schiek, H. Fang, R. Malendevich, and G. I. Stegeman, Phys. Rev. Lett. 86, 4528 (2001).

[55] M. Peccianti, C. Conti, and G. Assanto, Opt. Lett. 28, 2231 (2003).

[56] S. M. Sears, M. Soljacic, D. N. Christodoulides, and M. Segev, Phys. Rev. E 65, 036620 (2002).

[57] W. Krolikowski, O. Bang, J. J. Rasmussen, and J. Wyller, Phys. Rev. E 64, 016612 (2001).

[58] G. Assanto, M. Peccianti, and C. Conti, IEEE J. Sel. Top. Quantum Electron. 10, 862 (2004).

[59] D. Neshev, A. A. Sukhorukov, Y. S. Kivshar, and W. Krolikowski, Opt. Lett. 29, 2591 (2004).

[60] M. Peccianti, C. Conti, G. Assanto, A. D. Luca, and C. Umeton, Nature (London) 432, 733 (2004).

[61] S. Carrasco, S. Polyakov, H. Kim, L. Jankovic, G. I. Stegeman, J. P. Torres, L. Torner, M. Katz, and D. Eger, Phys. Rev. E 67, 046616 (2003).

[62] M. A. Porras, A. Parola, D. Faccio, A. Dubietis, and P. Di Trapani, Phys. Rev. Lett. 93, 153902 (2004).

[63] M. Centurion, Y. Pu, M. Tsang, and D. Psaltis, Phys. Rev. A 71, 063811 (2005).

[64] S. Skupin et al., Phys. Rev. E 70, 046602 (2004).

[65] M. Centurion, Y. Pu, and D. Psaltis, Opt. Express 13, 6202 (2005).

[66] L. Guyon, F. Courvoisier, V. Boutou, R. Nuter, A. Vinçotte, S. Champeaux, L. Bergé, P. Glorieux, and J. P. Wolf, Phys. Rev. A 73, 051802(R) (2006).

[67] A. Dubietis, E. Kucinskas, G. Tamosauskas, E. Gaizauskas, M. A. Porras, and P. DiTrapani, Opt. Lett. 29, 2893 (2004). 